\title{
Transcatheter Arterial Embolization as a Treatment for Medial Knee Pain in Patients with Mild to Moderate Osteoarthritis
}

\author{
Yuji Okuno • Amine Mohamed Korchi • \\ Takuma Shinjo • Shojiro Kato
}

Received: 16 February 2014/ Accepted: 20 May 2014/Published online: 4 July 2014

(C) Springer Science+Business Media New York and the Cardiovascular and Interventional Radiological Society of Europe (CIRSE) 2014

\begin{abstract}
Purpose Osteoarthritis is a common cause of pain and disability. Mild to moderate knee osteoarthritis that is resistant to nonsurgical options and not severe enough to warrant joint replacement represents a challenge in its management. On the basis of the hypothesis that neovessels and accompanying nerves are possible sources of pain, previous work demonstrated that transcatheter arterial embolization for chronic painful conditions resulted in excellent pain relief. We hypothesized that transcatheter arterial embolization can relieve pain associated with knee osteoarthritis.

Methods Transcatheter arterial embolization for mild to moderate knee osteoarthritis using imipenem/cilastatin sodium or $75 \mu \mathrm{m}$ calibrated Embozene microspheres as an embolic agent has been performed in 11 and three patients, respectively. We assessed adverse events and changes in
\end{abstract}

Y. Okuno $(\varangle) \cdot$ S. Kato

Department of Orthopedic Surgery, Edogawa Hospital, 2-24-18

Higashikoiwa, Edogawa-ku, Tokyo 133-0052, Japan

e-mail: how_lowlow@yahoo.co.jp

S. Kato

e-mail: shojiro7@yahoo.co.jp

A. M. Korchi

Department of Diagnostic and Interventional Radiology, Geneva University Hospitals, Rue Gabrielle-Perret-Gentil 4,

1211 Genève 14 , Switzerland

e-mail: amine.korchi@gmail.com

\section{T. Shinjo}

Institute for Integrated Sports Medicine, School of Medicine,

Keio University, 35 Shinanomachi, Shinjuku-ku,

Tokyo 160-8582, Japan

e-mail: shin.takuma@a7.keio.jp
Western Ontario and McMaster University Osteoarthritis Index (WOMAC) scores.

Results Abnormal neovessels were identified within soft tissue surrounding knee joint in all cases by arteriography. No major adverse events were related to the procedures. Transcatheter arterial embolization rapidly improved WOMAC pain scores from $12.2 \pm 1.9$ to $3.3 \pm 2.1$ at 1 month after the procedure, with further improvement at 4 months $(1.7 \pm 2.2)$ and WOMAC total scores from $47.3 \pm 5.8$ to $11.6 \pm 5.4$ at 1 month, and to $6.3 \pm 6.0$ at 4 months. These improvements were maintained in most cases at the final follow-up examination at a mean of $12 \pm 5$ months (range 4-19 months).

Conclusion Transcatheter arterial embolization for mild to moderate knee osteoarthritis was feasible, rapidly relieved resistant pain, and restored knee function.

Keywords Abnormal neovessels - Embolization · Osteoarthritis · Knee pain

\section{Introduction}

Osteoarthritis is a common and major cause of pain and disability. Disabling symptoms of knee osteoarthritis are seen in an approximately $10 \%$ of people over 55 years old [1].

The treatment armamentarium for knee osteoarthritis is broad and includes pharmacologic pain medications and major joint replacement surgery. Minor symptoms can be managed with pain relievers such as acetaminophen or nonsteroidal anti-inflammatory drugs. Severe and endstage osteoarthritis can be treated with total joint arthroplasty. However, moderate arthritis resistant to nonsurgical options, yet not severe enough to warrant joint replacement, represents a challenge in its management. For years, 
arthroscopic lavage and debridement have been performed for moderate osteoarthritis of the knee. However, compared to sham procedure, its effectiveness was not proven in a double-blinded, randomized, placebo-controlled trial [2]. Thus, there is still a need to develop a new, effective, minimally invasive and safe treatment for moderate osteoarthritis of the knee.

As a new treatment option for chronic musculoskeletal painful conditions, we previously reported a case series of transcatheter arterial embolization for refractory tendinopathy [3] and adhesive capsulitis [4]. This treatment is based on the notion that increased number of blood vessels and accompanying nerves are a possible source of chronic pain and that occlusion of these abnormal vessels might reduce such pain. We postulated that such a mechanism might also play an important role in knee osteoarthritis and that transcatheter arterial embolization of neovessels might relieve pain in this condition.

The purpose of the present study was to evaluate the feasibility and effects of transcatheter arterial embolization on chronic knee pain due to medial knee osteoarthritis refractory to traditional nonsurgical management.

\section{Materials and Methods}

Our institutional review board approved this prospective study, which was conducted between June 2012 and December 2013 in Edogawa Hospital (Tokyo, Japan). All patients received explanations about various management modalities and the potential risks, benefits, and outcomes of transcatheter arterial embolization as an alternative treatment for osteoarthritis related knee pain, and then provided written informed consent. All included patients had moderate to severe medial knee pain (visual analog scale [VAS] $>50 \mathrm{~mm}$ ) resistant to at least 3 months of conservative therapies (antiinflammatory drugs, physical therapy, muscle strengthening, and intra-articular injection of hyaluronic acid). All patients were assessed by routine radiographs, and those with severe osteoarthritis changes (Kellgren-Lawrence grade 3 or higher) were excluded because they were candidates for total knee arthroplasty. Other exclusion criteria were local infection, malignancy, advanced atherosclerosis, rheumatoid arthritis, and prior knee surgery. Nobody was lost to follow-up. Fourteen (eight female and six male subjects; mean age, $65.2 \pm 8.3$ years; range 49-76 years) patients were enrolled in the present study. Table 1 shows the baseline patients demographic and clinical data.

\section{Assessment}

The presence of knee pain and its relation with activity was noted. Routine knee examination was performed with
Table 1 Baseline characteristics of 14 patients

\begin{tabular}{|c|c|}
\hline Characteristics & Value \\
\hline Age $(y)$, mean \pm SD (range) & $65.2 \pm 8.3(49-76)$ \\
\hline Male gender & 6 \\
\hline Right-sided embolization & 5 \\
\hline Left-sided embolization & 9 \\
\hline Pain duration (mo), mean \pm SD (range) & $22.1 \pm 22.8(3-77)$ \\
\hline \multicolumn{2}{|l|}{ Kellgren-Lawrence grade } \\
\hline $0-1$ & 8 \\
\hline 2 & 6 \\
\hline BMI $\left(\mathrm{kg} / \mathrm{m}^{2}\right)$, mean $\pm \mathrm{SD}$ & $26.3 \pm 6.3$ \\
\hline VAS score $(\mathrm{mm})$, mean \pm SD & $70 \pm 5$ \\
\hline \multicolumn{2}{|l|}{ Previous treatment } \\
\hline Hyaluronic acid injection & 12 \\
\hline NSAID & 6 \\
\hline Physical therapy & 10 \\
\hline \multicolumn{2}{|l|}{ Tenderness } \\
\hline Over anteromedial aspect & 11 \\
\hline Over medial joint space & 9 \\
\hline Over anserine & 5 \\
\hline \multicolumn{2}{|l|}{ MRI findings } \\
\hline Synovial thickening or joint effusion & 12 \\
\hline Meniscus tear & 4 \\
\hline Abnormal intensity in infrapatellar fat pad & 7 \\
\hline
\end{tabular}

$\overline{B M I \text { body mass index, } V A S \text { visual analog scale, NSAID nonsteroidal }}$ anti-inflammatory drug, $M R I$ magnetic resonance imaging

particular emphasis on tenderness of the knee joint. In all patients, conventional radiography and MRI were performed. We used the Western Ontario and McMaster University Osteoarthritis Index (WOMAC) questionnaire, which includes 24 questions on daily activities. The WOMAC scoring system consists of three subscale scores for pain, stiffness, and physical function, resulting in a total WOMAC score. We used pain WOMAC scores and total WOMAC scores for evaluation. We also assessed overall pain using the VAS. Data from 11 patients treated with imipenem cilastatin sodium between June 2012 to January 2013 were reviewed at 1,4 , and 12 months after embolization. The other three patients treated with $75 \mu \mathrm{m}$ calibrated Embozene microspheres between August 2013 to September 2013 were reviewed at 1 and 4 months after embolization.

We assessed technical success and adverse events. Technical success was considered as being equivalent to the devascularization of a focal lesion or intentional reduction or cessation of blood flow to a target vascular bed or an entire organ. Major complications were considered to be those resulting in an unplanned increase in the level of care, prolonged hospitalization, permanent adverse sequelae, or death. We also assessed the postprocedural occurrence of 
Table 2 Comparison of two embolic materials

\begin{tabular}{lll}
\hline Characteristics & IPM/CS & Embozene \\
\hline Particle size & $10-70 \mu \mathrm{m}$ & $75 \mu \mathrm{m}$ (calibrated) \\
Embolic effect & Temporary & Longer term \\
Inflammatory reaction & Unknown & Low \\
Mean volume used in & $2.5 \mathrm{~mL} / 5 \mathrm{~mL}$ & $0.068 \mathrm{~mL} / 2 \mathrm{~mL}$ \\
this study & suspension & particle volume \\
\hline
\end{tabular}

IPM/CS imipenem/cilastatin sodium

tissue necrosis, dermal ulcer, tendon or ligament rupture, peripheral paresthesia, and other complications.

\section{Embolization Technique}

Under local anesthesia, percutaneous arterial access was obtained using a 3F introducer sheath (Super Sheath; Medikit Co. Ltd., Tokyo, Japan). The femoral artery was punctured in an ipsilateral anterograde fashion. After intravenous administration of 2,000 IU heparin (heparin sodium; Mitsubishi Tanabe Pharma Corporation, Osaka, Japan), a 3F angiographic catheter (Multipurpose; Medikit Co. Ltd., Tokyo, Japan) was introduced toward the popliteal artery. Digital subtraction angiography was then performed by manually injecting 3 to $5 \mathrm{~mL}$ of iodinated contrast medium (Hexabrix; Terumo, Tokyo, Japan). We precisely depicted the descending genicular artery, superior and inferior lateral genicular arteries, superior and inferior medial genicular arteries, and the median genicular artery in all patients. After having localized abnormal neovessels, a 2.4F microcatheter (Meister Cass; Medikit Co. Ltd., Tokyo, Japan) was inserted coaxially through the $3 \mathrm{~F}$ catheter and selectively placed in the targeted arteries (Table 2). Embolic agent was infused until blood flow stagnated. Hemostasis of the arterial access was achieved by manual compression for $10 \mathrm{~min}$ and bed rest for $2 \mathrm{~h}$ after the femoral sheath removal. The patients were discharged on the same day.

\section{Embolic Material}

We used imipenem/cilastatin sodium (IPM/CS) in 11 cases and $75 \mu \mathrm{m}$ Embozene microsphere in three cases. The characteristics of each agent are summarized in Table 2. The IPM/CS embolic agent was selected on the basis of a previous report of transcatheter arterial embolization of refractory painful tendinopathy and enthesopathy [3]. This agent is approved as an antibiotic, is slightly soluble in water, and, when suspended in contrast agent, forms 10 to $70 \mu \mathrm{m}$ particles that exert an embolic effect $[5,6]$. Aihara [5] reported that a suspension of IPM/CS in contrast agent has caused microembolization of blood vessels smaller than $60 \mu \mathrm{m}$ in diameter within tumor tissue transplanted into rabbits. In addition, Woodhams et al. [6] performed transcatheter arterial embolization by IPM/CS to treat intestinal bleeding and reported that hemostasis was obtained successfully. In the present study, a suspension of $0.5 \mathrm{~g}$ of IPM/CS (Primaxin; Merck \& Co. Inc., Whitehouse Station, NJ, USA) in $5 \mathrm{~mL}$ of iodinated contrast agent was prepared by pumping syringes for $10 \mathrm{~s}$, and then injected in $0.2 \mathrm{~mL}$ increments until blood flow stagnated.

For $75 \mu \mathrm{m}$ Embozene microspheres, $0.15 \mathrm{~mL}$ of that solution was diluted with $2 \mathrm{~mL}$ of contrast agent and injected in $0.2 \mathrm{~mL}$ increments until blood flow stagnated.

\section{Postprocedural Follow-up and Therapy}

We reviewed the postprocedural occurrence of tissue necrosis, dermal ulcer, tendon or ligament rupture, peripheral paresthesia, and other complications. Patients could continue with previous therapies and resume activities freely from the day after treatment, but they were not allowed to undergo any new, additional, or concomitant therapy until final follow-up.

\section{Statistical Analysis}

Baseline and outcome variables were compared by Dunnett's post hoc test to determine changes in WOMAC scores before the procedure and at 1, 4, and 12 months after the procedure. All data were statistically analyzed by SPSS software, version 11.0 (IBM, Armonk, NY, USA). A $p$ value of $<0.05$ was considered statistically significant.

\section{Results}

The technical success rate was $100 \%$. Figures $1,2,3$, and 4 show examples of digital subtraction angiography images and MRI. Abnormal neovessels were clearly depicted in all patients, originating from several arteries, including descending genicular artery $(n=13$, Figs. $1,2,3,4)$, inferior medial genicular artery $(n=8$, Fig. 2$)$, inferior lateral genicular artery $(n=7)$, superior lateral genicular artery $(n=2)$, median genicular artery $(n=2)$, and superior medial genicular artery $(n=1)$. In all patients, the abnormal neovessels were located in the painful area of the knee. No major adverse events were related to the procedures in patients treated with IPM/CS and Embozene. Moderate subcutaneous hemorrhage at the puncture site in one patient resolved within 1 week. Tissue necrosis, dermal ulcer, tendon or ligament rupture, and peripheral paresthesia did not arise in any embolized territory.

The mean WOMAC pain score of all treated patients significantly decreased from $12.2 \pm 1.9$ to $3.3 \pm 2.1$ at 


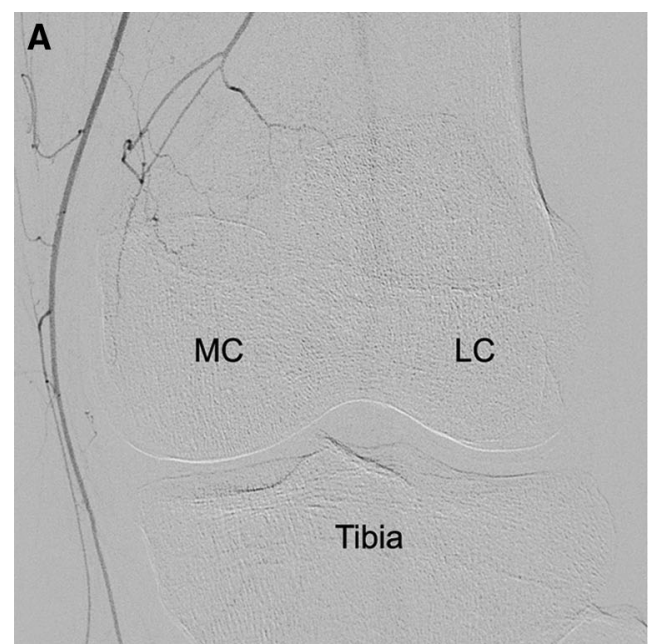

Fig. 1 Normal and abnormal vasculature of descending genicular artery. Angiographic findings of left descending genicular artery in normal knee (A) and from a 49-year-old man with periosteum and

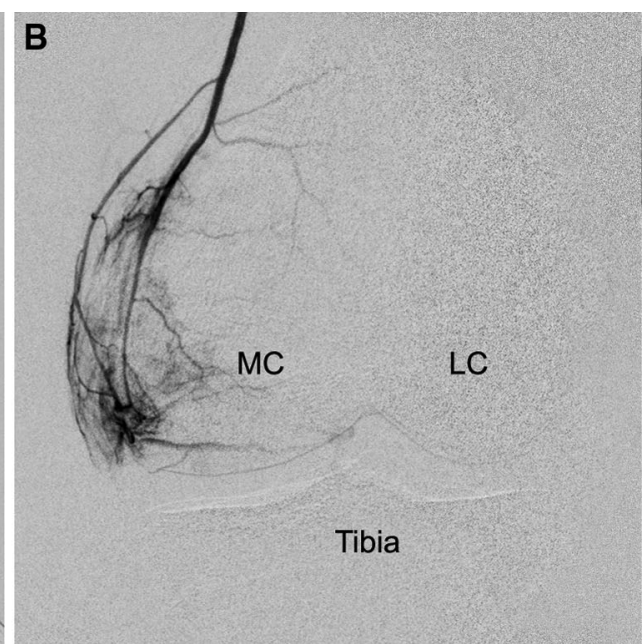

synovial thickening in left knee (B). Abnormal neovessels adjacent to medial condyle were confirmed. $M C$ medial condyle, $L C$ lateral condyle
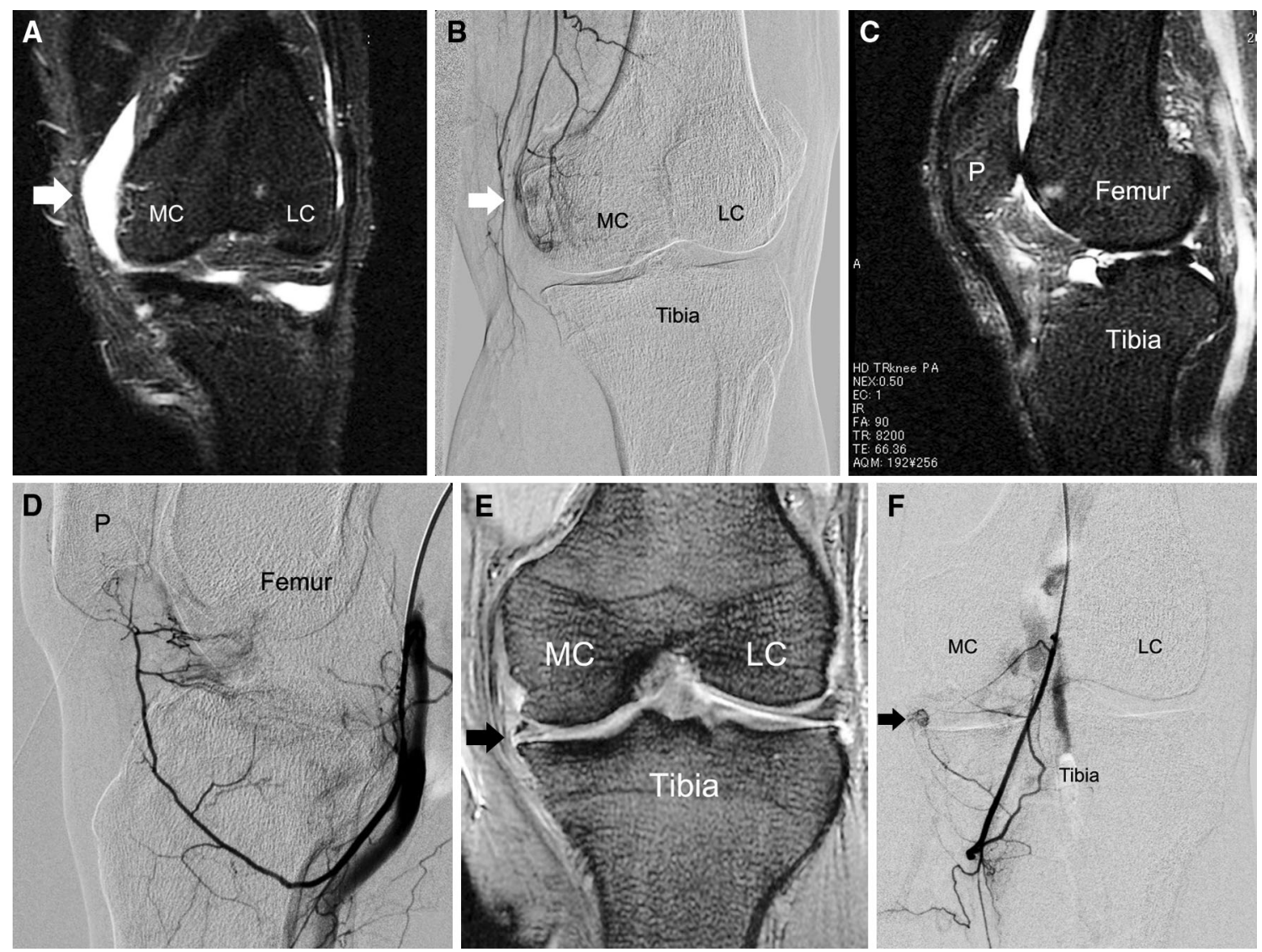

Fig. 2 MRI findings and angiographic findings in a 70-year-old patient with moderate osteoarthritis. Coronal STIR MRI (A) shows effusion around medial condyle (white arrow). Abnormal neovessels (white arrow, B) of same region was confirmed by selective angiography of left descending genicular artery (B). Sagittal STIR MRI (C) shows abnormal high intensity in infrapatellar region. Abnormal neovessels in infrapatellar region was confirmed from lateral view of inferior medial genicular artery (D). Coronal gradient recalled echo T2-weighted image (E) shows torn medial meniscus (black arrow). Abnormal neovessels at meniscus base (black arrow, F) were confirmed from inferior medial genicular artery $(\mathbf{F})$. STIR short tau inversion recovery, $M C$ medial condyle, $L C$ lateral condyle, $P$ patellar 

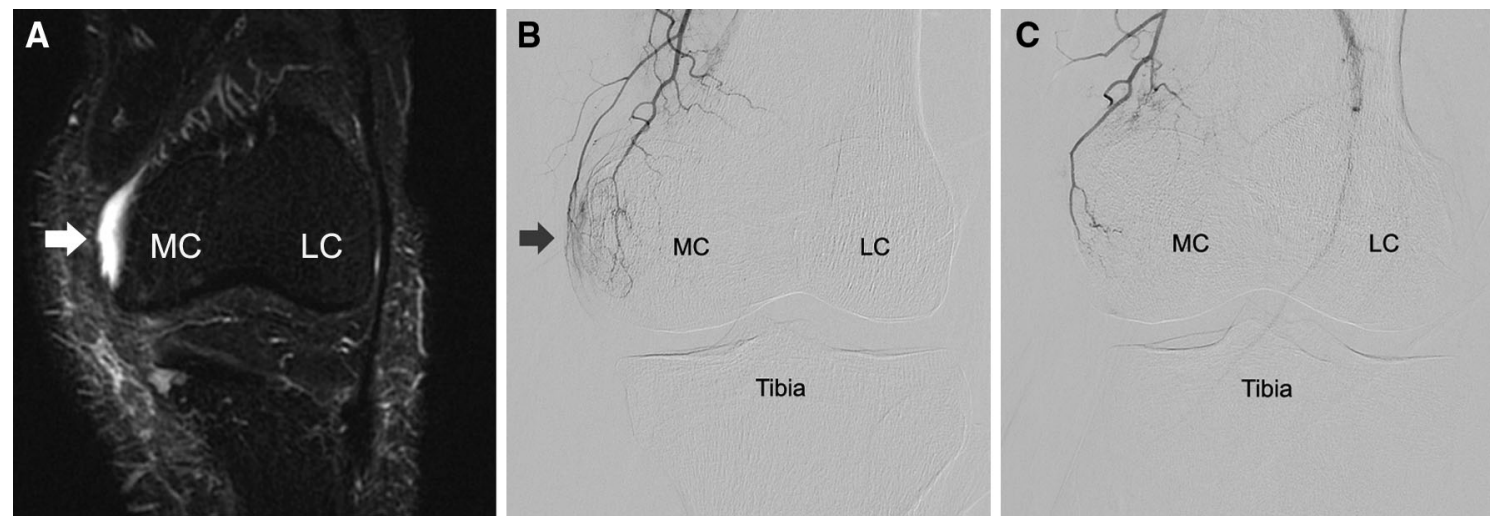

Fig. 3 Angiographic findings before and after transcatheter arterial embolization in a 61-year-old patient treated with IPM/CS. Coronal STIR MRI (A) showing joint effusion (white arrow, A) and angiographic comparison before (B) and after (C) embolization. B Selective angiography from descending genicular artery before embolization shows abnormal neovessels (black arrow) adjacent to medial condyle. C Postembolization angiogram shows elimination of hypervascularity. IPM/CS imipenem/cilastatin sodium, STIR short tau inversion recovery, $M C$ medial condyle, $L C$ lateral condyle

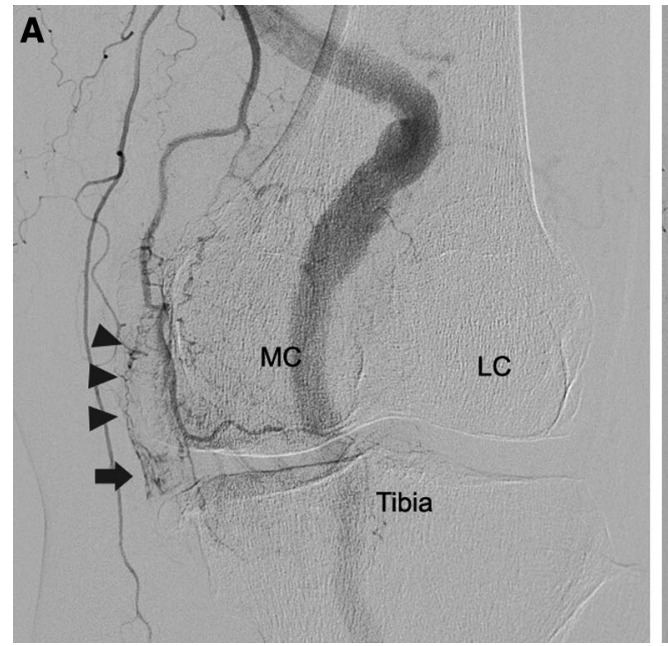

Fig. 4 Angiographic findings before and after transcatheter arterial embolization in a 72-year-old woman treated with microsphere Embozene. Left descending genicular artery before $(\mathbf{A})$ and after (B) embolization. Abnormal neovessels confirmed at meniscus base

1 month after the procedure, with further improvement at 4 months $(1.7 \pm 2.2)$, and the mean WOMAC total score decreased from $47.3 \pm 5.8$ to $11.6 \pm 5.4$ at 1 month, and to $6.3 \pm 6.0$ at 4 months (all $p<0.001$ ). These improvements were maintained in most cases at the final follow-up examination at a mean of $12 \pm 5$ months (range 4-19 months) (Table 3). The mean overall VAS scores before treatment significantly decreased at 1 week and at 1 and 4 months thereafter $(70 \pm 5$ vs. $29 \pm 17,21 \pm 16$, and $13 \pm 15 \mathrm{~mm}$ respectively; all $p<0.001)$. The dose of medication and the frequency of injection therapy decreased after procedure (Table 3 ).

The change of pain WOMAC score, total WOMAC score, pain symptoms frequency, and number of patients

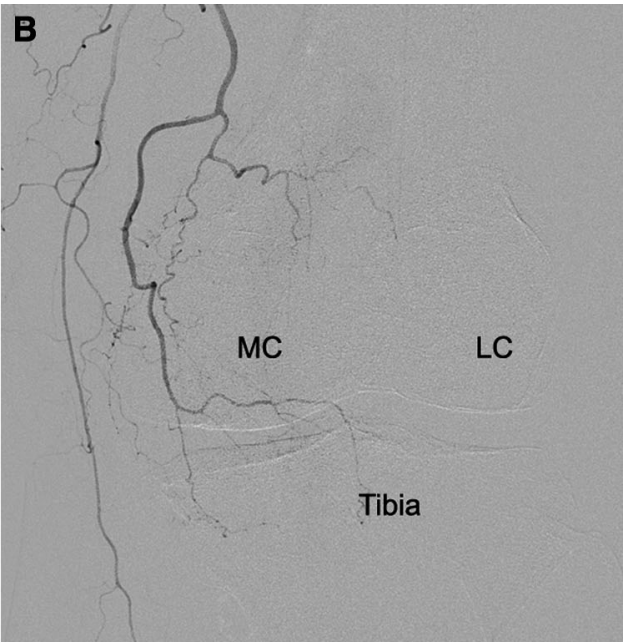

(arrow) and medial side of joint capsule (arrowhead). B Postembolization angiogram shows elimination of hypervascularity. $M C$ medial condyle, $L C$ lateral condyle

who used other therapies in each treatment groups are summarized in Table 3.

\section{Discussion}

In the present study, all patients with symptomatic medial knee osteoarthritis had abnormal neovessels located within periarticular soft tissues. Transcatheter arterial embolization using either IPM/CS suspension or $75 \mu \mathrm{m}$ calibrated Embozene microspheres could relieve pain symptoms and improve functional scores.

The cause of pain in knee osteoarthritis remains elusive as the primary site of pathology, cartilage, has no pain 
Table 3 Changes in clinical features and WOMAC scores throughout the study

\begin{tabular}{|c|c|c|c|c|}
\hline Characteristics & Baseline & 1 Month & 4 Months & 12 Months \\
\hline \multicolumn{5}{|l|}{ IPM/CS group $(n=11[5])$} \\
\hline Pain during walking & $8[4]$ & $2[2]$ & $1[1]$ & $1[1]$ \\
\hline Pain using stairs & $11[5]$ & $4[2]$ & $1[1]$ & $2[1]$ \\
\hline Pain WOMAC, mean \pm SD & $12.1 \pm 1.8$ & $3.5 \pm 2.4$ & $1.8 \pm 2.5$ & $1.9 \pm 2.7$ \\
\hline Total WOMAC, mean \pm SD & $48.5 \pm 9.4$ & $12.5 \pm 7.6$ & $7.5 \pm 6.4$ & $6.0 \pm 8.3$ \\
\hline Patients receiving NSAIDs & 8 & 3 & 1 & 1 \\
\hline Patients receiving HA inj & 5 & 0 & 0 & 1 \\
\hline \multicolumn{5}{|l|}{ Embozene group $(n=3[1])$} \\
\hline Pain during walking & $3[1]$ & 0 & 0 & \\
\hline Pain using stairs & $3[1]$ & $1[1]$ & 0 & \\
\hline Pain WOMAC, mean \pm SD & $12.6 \pm 2.5$ & $2.7 \pm 1.2$ & $1.3 \pm 1.1$ & \\
\hline Total WOMAC, mean \pm SD & $43.3 \pm 6.8$ & $9.0 \pm 4.6$ & $6.0 \pm 5.3$ & \\
\hline Patients receiving NSAIDs & 2 & 1 & 0 & \\
\hline Patients receiving HA injection & 1 & 0 & 0 & \\
\hline
\end{tabular}

Numbers in square brackets indicate number of patients with Kellgren-Lawrence grade 2 radiographic change

WOMAC Western Ontario and McMaster University Osteoarthritis Index, IPM/CS imipenem/cilastatin sodium, NSAID nonsteroidal antiinflammatory drug, $H A$ inj intra-articular hyaluronic acid injection

nerve fibers. Moreover, many studies have reported a high degree of discordance between clinical symptoms and radiographic findings of knee osteoarthritis [7-9]. In our study, patients with mild or minimal degenerative changes and severe symptoms were significantly relieved by embolization treatment. In addition, excellent pain relief was seen in patients with Kellgren-Lawrence grade 2 degenerative changes. These findings indicate that pain in osteoarthritis does not necessarily come from the degenerative site or from cartilage loss.

It is becoming increasingly apparent that the periarticular soft tissues, such as synovium, fat pad, periosteum, and joint capsule, are the likely source of nociception in knee joint [10]. In a study including patients with the same radiographic grade of knee osteoarthritis, the synovial thickening and effusion over the medial knee recess (depicted on ultrasound) was significantly associated with medial knee pain after adjustment [11]. In the study of Crema et al. [12], abnormal contrast enhancement observed on MRI in the infrapatellar fat pad and peripatellar soft tissues was significantly associated with pain in the fully adjusted model. Angiographic findings in the present study revealed abnormal neovessels within several periarticular tissues, including synovium and periosteum around the medial condyle (Figs. 1, 2, 3), infrapatellar fat pad (Fig. 2D), medial meniscus base (Figs. 2F, 4), and medial side of joint capsule (Fig. 4). Pain relief achieved by occluding these abnormal neovessels indicated that these abnormal neovessels within the periarticular soft tissue play an important role in pain symptoms.

Further mechanisms by which transcatheter arterial embolization relieves patients' symptoms remain obscure.
However, focusing on the time course of pain and symptoms relief might provide us with some insights about the rationale of this treatment. From our experiences, there were two distinct time points when pain and symptoms improved. One was soon after embolization; patients stated that their tenderness decreased few minutes after infusion of the IPM/CS suspension or $75 \mu \mathrm{m}$ calibrated Embozene. The other occurred later-several weeks or months after embolization. One possible mechanism of the first immediate improvement is that decreasing abnormal blood flow somehow reduced the accompanying sensory nerve stimulation. It is already known that inflammation leads to angiogenesis and sensory nerve growth along new blood vessels in osteoarthritic joints [13]. In the immunohistochemical analysis, neovessels and accompanying nerve growth have been reported in synovium [14] and fat pads $[15,16]$ of osteoarthritic joints. However, to our knowledge, nobody has clearly demonstrated that these sensory nerves along vessels play an important role in patients' symptoms. Thus, further basic experiments would be required.

The later onset of improvement demonstrated in the follow-up period may indicate that occluding neovessels contributed to the suppression of inflammation. Lyu et al. [17] prospectively analyzed the macroscopic appearance and histological features of removed medial synovium from 48 consecutive patients who underwent total knee replacement for severe medial compartment osteoarthritis; they found that large amount of proliferative vessels and lymphoplasmacytic cells had infiltrated in the pathological synovial tissue. Transcatheter arterial embolization might act by stopping the influx of inflammatory cells in 
synovial tissues and thus have a beneficial effect against inflammation and pain.

Joint hypervascularization is included indirectly in current scoring systems for osteoarthritis by assessing the degree of synovitis using Doppler ultrasound and/or MRI [18-20]. Direct evaluation of neohypervascularization is not included in the current scoring systems, and its assessment in osteoarthritic patients by arteriography does not appear to be a reasonable option as a result of its relative invasiveness compared to other diagnostic imaging modalities. However, angio-MRI sequences with high spatial and temporal resolution could offer an alternative to analyze the extent and topography of neovascularization. As demonstrated by Hash et al. [21] in a series of 18 patients with recurrent hemarthrosis after total knee replacement, angio-MRI appeared to be a useful noninvasive tool to assess the vascular anatomy and to depict the artery supplying the hypervascular synovium causing hemarthrosis. In this case series [21], MRI allowed the identification of candidate patient for transcatheter arterial embolization. We did not perform magnetic resonance angiography in our study, but this could be incorporated in further studies in order to evaluate its performance in selecting candidates for potential pain-relieving embolization.

We used two different types of embolic agent in the present study, IPM/CS particles and $75 \mu \mathrm{m}$ calibrated Embozene, and both effectively relieved medial knee pain without major complications. However, it is hard to demonstrate which particle is more effective as a result of the small patient cohort. Furthermore, the true incidence of complications of each technique could not be meaningfully determined in the present study. Thus, further studies and more experience are required to determine optimal treatment.

The present study has several limitations. We had no control group, and the patients were not blinded to their treatment. After the procedure, patients in the present study could continue the therapies used before embolization, and this could represent a bias in our results. Finally, the patient cohort was small and the follow-up period short. Thus, further studies are needed to validate our procedure.

In conclusion, transcatheter arterial embolization targeting abnormal neovascularization in osteoarthritis is a feasible and safe interventional radiology procedure, and it effectively relieves medial knee pain due to moderate osteoarthritis refractory to traditional nonsurgical management.

Conflict of interest Yuji Okuno, Amine M. Korchi, Takuma Shinjo, and Shojiro Kato declare that they have no conflict of interest.

Funding None

\section{References}

1. Peat G, McCarney R, Croft P (2001) Knee pain and osteoarthritis in older adults: a review of community burden and current use of primary health care. Ann Rheum Dis 60:91-97

2. Moseley JB, O'Malley K, Petersen NJ et al (2002) A controlled trial of arthroscopic surgery for osteoarthritis of the knee. N Engl J Med 347:81-88

3. Okuno Y, Matsumura N, Oguro S (2013) Transcatheter arterial embolization using imipenem/cilastatin sodium for tendinopathy and enthesopathy refractory to nonsurgical management. J Vasc Interv Radiol 24:787-792

4. Okuno Y, Oguro S, Iwamoto W et al (2014) Short-term results of transcatheter arterial embolization for abnormal neovessels in patients with adhesive capsulitis: a pilot study. J Shoulder Elbow Surg. doi:10.1016/j.jse.2013.12.014

5. Aihara T (1999) A Basic study of super-selective transcatheter arterial chemotherapy and chemoembolization. (1). Establishment of an animal model for super-selective transcatheter arterial chemotherapy and preparation for appro-priate suspension of microembolization. Kawasaki Igakkaishi 25:47-54

6. Woodhams R, Nishimaki H, Ogasawara G et al (2013) Imipenem/ cilastatin sodium (IPM/CS) as an embolic agent for transcatheter arterial embolisation: a preliminary clinical study of gastrointestinal bleeding from neoplasms. Springerplus 2:344

7. Hart DJ, Spector TD, Brown P et al (1991) Clinical signs of early osteoarthritis: reproducibility and relation to $\mathrm{X}$ ray changes in 541 women in the general population. Ann Rheum Dis 50:467-470

8. Bedson J, Croft PR (2008) The discordance between clinical and radiographic knee osteoarthritis: a systematic search and summary of the literature. BMC Musculoskelet Disord 9:116

9. Claessens AA, Schouten JS, van den Ouweland FA, Valkenburg HA (1990) Do clinical findings associate with radiographic osteoarthritis of the knee? Ann Rheum Dis 49:771-774

10. Dye SF, Vaupel GL, Dye CC (1998) Conscious neurosensory mapping of the internal structures of the human knee without intraarticular anesthesia. Am J Sports Med 26:773-777

11. Wu PT, Shao CJ, Wu KC et al (2012) Pain in patients with equal radiographic grades of osteoarthritis in both knees: the value of gray scale ultrasound. Osteoarthritis Cartilage 20:1507-1513

12. Crema MD, Felson DT, Roemer FW et al (2013) Peripatellar synovitis: comparison between non-contrast-enhanced and contrast-enhanced MRI and association with pain. The MOST study. Osteoarthritis Cartilage 21:413-418

13. Mapp PI, Walsh DA (2012) Mechanisms and targets of angiogenesis and nerve growth in osteoarthritis. Nat Rev Rheumatol 8:390-398

14. Walsh DA, Bonnet CS, Turner EL et al (2007) Angiogenesis in the synovium and at the osteochondral junction in osteoarthritis. Osteoarthritis Cartilage 15:743-751

15. Gardner E (1948) The innervation of the knee joint. Anat Rec 101:109-130

16. Bohnsack M, Meier F, Walter GF et al (2005) Distribution of substance-P nerves inside the infrapatellar fat pad and the adjacent synovial tissue: a neurohistological approach to anterior knee pain syndrome. Arch Orthop Trauma Surg 125:592-597

17. Lyu SR, Chiang JK, Tseng CE (2010) Medial plica in patients with knee osteoarthritis: a histomorphological study. Knee Surg Sports Traumatol Arthrosc 18:769-776

18. Guermazi A, Hayashi D, Eckstein F et al (2013) Imaging of osteoarthritis. Rheum Dis Clin North Am 39:67-105

19. Walther M, Harms H, Krenn V et al (2002) Synovial tissue of the hip at power Doppler US: correlation between vascularity and power Doppler US signal. Radiology 225:225-231 
20. Walther M, Harms H, Krenn V et al (2001) Correlation of power Doppler sonography with vascularity of the synovial tissue of the knee joint in patients with osteoarthritis and rheumatoid arthritis. Arthritis Rheum 44:331-338
21. Hash TW 2nd, Maderazo AB, Haas SB et al (2011) Magnetic resonance angiography in the management of recurrent hemarthrosis after total knee arthroplasty. J Arthroplasty 26(1357-1361):e1 